\title{
La gestion environnementale des sports de nature : entre laisser-faire, autorité et concertation
}

Jean-Pierre Mounet

\section{(2) OpenEdition}

Journals

Édition électronique

URL : https://journals.openedition.org/developpementdurable/3817

DOI : 10.4000/developpementdurable.3817

ISSN : 1772-9971

Éditeur

Association DD\&T

Référence électronique

Jean-Pierre Mounet, «La gestion environnementale des sports de nature : entre laisser-faire, autorité et concertation », Développement durable et territoires [En ligne], Varia (2004-2010), mis en ligne le 11 juin 2007, consulté le 21 septembre 2021. URL : http://journals.openedition.org/

developpementdurable/3817 ; DOI : https://doi.org/10.4000/developpementdurable.3817

Ce document a été généré automatiquement le 21 septembre 2021.

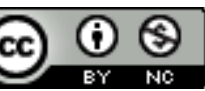

Développement Durable et Territoires est mis à disposition selon les termes de la licence Creative Commons Attribution - Pas d'Utilisation Commerciale 4.0 International. 


\title{
La gestion environnementale des sports de nature : entre laisser-faire, autorité et concertation
}

\author{
Jean-Pierre Mounet
}

1 Cet article interroge les conséquences de la croissance des sports de nature dans leur rapport à l'environnement. Leur évolution récente pose le problème de leur durabilité vue sous l'angle de la préservation environnementale au sein d'espaces protégés comme de la nature ordinaire. En effet, la protection de la nature, qui est le support de ces activités, n'est pas le point le plus fort des politiques environnementales (Kalaora, 1998). Cette durabilité ne concerne pas les aménagements ou les équipements, rares au demeurant, qui relèvent de dispositions réglementaires comme, par exemple, une notice d'impact, mais plutôt l'investissement léger et diffus qu'ils opèrent dans le milieu naturel. A l'heure où les sites de nature commencent à être en quelque sorte institutionnalisés au niveau local sous la forme d'un plan départemental, il est intéressant d'analyser comment des activités en émergence ont pu faire l'objet de tentatives de gestion environnementale encore très dispersées.

2 Dans la nature ordinaire, les sports de nature se sont développés pratiquement sans autres contraintes que celles liées aux conflits d'usages et la prise en compte de l'environnement ne relevait donc que d'une sensibilité des pratiquants sportifs dont les compétences environnementales sont généralement réduites. Ainsi beaucoup de sportifs considèrent-ils encore que laisser les lieux propres suffit à protéger l'environnement.

Lorsque l'impact des sports de nature a commencé à être mis en cause, les réactions de leurs pratiquants ont été variées : quelques très rares fédérations et syndicats sportifs ont immédiatement tenté d'analyser le problème et d'en tenir compte. Mais, dans la plupart des autres cas, ce sont des acteurs extérieurs aux activités concernées qui se sont faits les porte-parole de l'environnement. En effet, les pratiquants sportifs, et particulièrement leurs porte-parole fédéraux, ont perçu le souci environnemental principalement comme une contrainte et un risque de se voir refuser l'accès aux sites de pratique. 
4 Les conflits d'usage se sont donc doublés de conflits environnementaux avec, parfois, une certaine ambiguïté. Car, au-delà de l'aspect purement biologique, l'impact environnemental des sports de nature a aussi été mobilisé dans les conflits d'usage comme un argument, parfois non fondé, par les opposants aux sports de nature sous la forme d'une "montée en généralité » visant à "masquer l'intérêt particulier derrière une contribution au bien commun» (Beuret, 2006, 165-166). Et il est donc parfois difficile de savoir a priori si certains conflits étaient liés à des usages concurrentiels des lieux ou à la protection de l'environnement.

Pour répondre à ces situations difficiles et tenter de les réguler, des décisions locales ont donc été prises sous la pression d'acteurs opposés à ces activités sous la forme de divers textes locaux, arrêtés municipaux, préfectoraux et de protection de biotope. Cependant, en marge de ces contextes turbulents, et probablement en réponse à leurs conséquences, de multiples situations expérimentales ont vu le jour dans lesquelles la régulation de ces activités est le fait d'acteurs divers. Seuls des exemples ayant un lien avec l'environnement seront évoqués bien que d'autres préoccupations (comme la sécurité) puissent prendre le pas sur celui-ci.

6 Il s'agit ici de s'interroger sur les modalités des gestions environnementales des sports de nature engagées de façon expérimentale et essentiellement dans des espaces protégés ainsi que sur leur efficacité. Il existe actuellement une ambiguïté des politiques environnementales soulignée par Theys (2003) : elles peuvent prendre la forme d'une gestion autoritaire et administrative ou tenter d'établir une concertation et une gouvernance environnementale.

7 Dans le cas des sports de nature, l'existence d'une controverse scientifique rend difficilement acceptable pour les acteurs de se voir imposer une décision autoritaire. De plus la protection de l'environnement apporte beaucoup plus de contraintes que d'avantages aux acteurs sportifs bien qu'ils évoquent souvent le fait que la nature est « leur fond de commerce ». En effet, la plupart d'entre eux la pratiquent plutôt comme un «terrain de jeu » et malgré une évolution encore très récente, rares sont encore les sportifs qui s'intéressent vraiment à l'aspect naturaliste de celui-ci, à l'exception des accompagnateurs de montagne qui en ont fait une part de leur activité.

8 L'aspect participatif et concerté peut donner lieu à diverses définitions selon les auteurs, et la concertation, portée par la tendance actuelle, peut être liée à diverses situations : controverses, crise difficile à résoudre, etc. Par exemple, Callon et al (2003) expliquent comment peut se construire un "monde commun», Beuret (2006) oppose les décisions selon qu'elles sont le fruit d'interactions verticales ou horizontales, Mermet (1992) voit dans la gestion communautaire un moyen de substituer la gestion intentionnelle à une gestion effective et indirecte dans un processus actif de communication et d'échanges, ou encore Theys (2003) considère que la gouvernance environnementale répond à des interactions non hiérarchiques et à la recherche de solutions acceptables pour les acteurs concernés. Tous ces auteurs s'accordent, a minima, sur l'aspect de construction collective des problèmes et des solutions ainsi que la recherche de solutions de type "gagnant gagnant » : nous retiendrons donc ces éléments pour qualifier la gestion concertée. Dans la gestion environnementale des sports de nature, il est intéressant d'interroger la confrontation entre application du cadre juridique et concertation, autrement dit entre autorité légale et "pouvoir », au sens que lui donne Dahl (1957), c'est-à-dire la capacité pour un acteur de modifier par ses actions le comportement d'un autre acteur. Cette ambiguïté entre autorité et pouvoir est d'autant plus intéressante que la plupart des 
exemples sont pris dans des espaces protégés. Jusqu'à quel point une contractualisation se développe-t-elle dans les espaces protégés disposant ou non de prérogatives réglementaires fortes? Comment ces tendances actuelles se combinent-elles avec l'autorité légitime détenue par les Parcs nationaux et les Réserves naturelles, avec la contractualisation qui caractérise les Parcs naturels régionaux ou encore avec l'absence de gestionnaire dans la nature ordinaire ? Les exemples seront tout d'abord pris dans des espaces protégés à prérogatives réglementaires fortes (Réserves naturelles et Parcs nationaux), puis un complément d'analyse évoquera des situations dans des Parcs naturels régionaux et dans la nature ordinaire.

Les sports de nature

9 Les sports de nature sont très divers. En effet, c'est plutôt leur hétérogénéité qui les caractérise que ce soit en terme d'aménagement, d'équipement, de niveau de risque, de technicité, d'engagement personnel et de capacité physique. Il suffit pour en être persuadé d'éclairer la comparaison par quelques exemples : randonnée pédestre, VTT, canyoning, escalade, sports d'eau vive, voile, spéléologie, etc. La pratique de ces activités de nature a souvent commencé il y a bien longtemps et sous une forme utilitaire comme en témoigne l'histoire de la raquette à neige ou celle des diverses embarcations de loisir. Puis, quelques rares pratiquants sportifs pionniers se sont aventurés dans le milieu naturel, parfois depuis plus d'un siècle, (par exemple, la « varappe » a débuté vers 1880 en forêt de Fontainebleau, Kalaora, 1993).

10 Ce n'est qu'au tournant des années soixante-dix et quatre-vingt que ces activités jusque-là très discrètes ont brusquement connu une forte démocratisation, puis une réelle massification. Les quelques pratiquants totalement autonomes et ne supportant pas des densités élevées sur les sites qu'ils fréquentaient (Becker et al, 1980) ont d'abord été rejoints par des néophytes souvent formés dans des clubs sportifs, puis par les clients des prestataires sportifs. Ces derniers étaient eux-mêmes souvent issus des rangs des pionniers et désiraient pouvoir vivre de leur passion. De plus, la multiplication des balisages et des topo-guides a ouvert le milieu naturel à de nouvelles catégories de publics. La massification a donc été le fait d'un changement de public et de logique de développement: à l'autonomie des pionniers s'est substituée celle apportée par l'encadrement sportif et par la sécurisation du milieu. L'offre commerciale en sports de nature est bientôt devenue indispensable pour conforter celle des destinations touristiques (Mounet, 1997) ce qui leur confère une légitimité économique. Parallèlement à ce phénomène, l'aspect compétitif s'est également développé avec ses exigences particulières : stades de slalom sur neige et en rivière, murs d'escalades, régates, raids de toutes sortes, etc. Pour compléter le tableau, de très nombreuses activités nouvelles ont brusquement vu le jour et ce phénomène existe toujours actuellement (Pociello, 1981 et 1995, Vigarello, 1981).

11 Les conséquences de cette évolution ont été de trois sortes. D'une part, des flux importants sont apparus là où, naguère, ne venaient que quelques initiés. D'autre part, les sites utilisés par les sports de nature se sont multipliés. Enfin, les demandes de facilitation et de sécurisation, cette dernière relayée notamment par des élus locaux inquiets de la possible mise en cause de leur responsabilité, ont induit une forte pression d'équipement et d'aménagement du milieu dont la station de ski alpin représente l'archétype.

12 L'image traditionnellement « écolo » des sports de nature s'est alors en partie effacée pour certains acteurs avec une mise en cause de leur impact environnemental d'autant plus forte que ces activités étaient plus nouvelles ou renouvelées dans leurs modalités. 
Parallèlement, de forts conflits d'usages avec les acteurs traditionnels du milieu rural (agriculteurs, éleveurs, chasseurs, pêcheurs, propriétaires ou occupants locaux) ont marqué les années quatre-vingt-dix en raison de la gêne induite par ces nouveaux flux parfois très importants. L'opposition a été particulièrement forte avec les chasseurs et les pêcheurs dont l'activité demande calme et silence : dès 1980, Jacob et Schreyer avaient déjà décrit ce type d'opposition entre des modes opposés de rapport à l'environnement, focalisé pour ces deux activités traditionnelles et non focalisé pour la presque totalité des sportifs. Des tentatives d'exclusion des sites de pratiques de ces protagonistes gênants, allant parfois jusqu'à la confrontation physique, ont alors eu lieu conformément à ce que Mac Ivy et al (1992) ont décrit dans des situations analogues.

Le jeu des acteurs

Les sites utilisés par les sports de nature concernent tous les milieux, depuis les littoraux jusqu'aux montagnes élevées en passant par les milieux aquatiques, aériens, neigeux et souterrains. Contrairement aux stades, gymnases, piscines, patinoires, etc., ces lieux ne sont pas dédiés à l'origine aux activités sportives mais sont utilisés par d'autres activités humaines, productives, résidentielles ou de loisir ou encore font l'objet d'autres objectifs comme la protection de la nature. La nouveauté sociale de ces activités s'est accompagnée d'une absence d'encadrement juridique autre que purement sportif, ce dernier ne concernant en rien les sites locaux de pratique. Pour ceux-ci n'existaient, à l'origine, que des lois et règlements adaptés à d'autres usages, à deux exceptions près cependant, le ski et la randonnée pédestre ${ }^{1}$ en raison de leur antériorité.

Les utilisateurs antérieurs des lieux ont été enrôlés bien malgré eux par le "problème commun» (Friedberg, 1993) de la présence de ces nouveaux arrivés. Ils se sont donc positionnés avec les sportifs en «coopération conflictuelle » autour du "problème» de leur coexistence en de mêmes lieux. A l'inverse de la prise en compte simultanée des trois pôles - social, économique et environnemental - préconisée dans le développement durable, chaque groupe a défendu ses seuls intérêts au détriment de ceux de ses protagonistes, dans des jeux à somme nulle où ce que gagne l'un est perdu par l'autre.

Ceci a déclenché une effervescence sociale parfois intense où conflits et coopérations ont structuré des contextes d'action encore non formalisés et la contingence des collectifs constitués a largement alimenté l'originalité des situations locales. Peu à peu des formalisations prenant des formes très diverses - ententes de gré à gré implicites ou explicites, chartes, arrêtés municipaux et préfectoraux - ont cependant permis de stabiliser les situations. Puis, la loi a progressivement entériné la présence de ces nouvelles activités. Mais il faudra attendre la loi sur le sport du 6 juillet 2000 pour que soit enfin envisagée la création d'un Plan départemental des espaces, sites et itinéraires relatifs aux sports de nature (PDESI) sous l'autorité du président du conseil général et avec l'appui d'une commission départementale de même nom (CDESI) au rôle délibératif. Ce nouveau texte, qui permet en quelque sorte d'institutionnaliser les sites sportifs de nature au niveau local en leur apportant une garantie de pérennité, inclut également une prise en compte de l'environnement mais sans en préciser les modalités.

Un impact environnemental complexe et controversé

L'impact environnemental des sports de nature a donné lieu à une abondante littérature de valeur très inégale. Une analyse bibliographique de 300 documents (Mounet et al, 2004) fondée sur une grille d'évaluation de ces études d'impact (Mounet et al, 2000), a mis en évidence les lacunes importantes de la plupart d'entre eux. Il est, en effet, difficile, pour des activités considérées encore comme marginales, de susciter un financement 
suffisant pour réaliser des analyses complexes et nécessitant un important suivi dans le temps. En France, de nombreuses études ont ainsi été confiées à des étudiants stagiaires avec bien peu de moyens tandis que les Parcs Nord-Américains ont piloté quelques programmes de recherche appliquée sur ce thème.

Dans ce contexte, la plupart des études traitent d'espèces emblématiques, abordent assez peu la dynamique des populations et ignorent généralement l'aspect écosystémique. Du fait de la complexité des problèmes abordés, et sauf impact massif et évident, même les études les plus sérieuses ont le plus grand mal à établir la réalité des impacts qui restent donc potentiels : ces impacts potentiels, par nature hypothétiques, ne peuvent pourtant être considérés ni comme inexistants, ni comme prouvés. L'incertitude scientifique qui en résulte, et qui est aussi le fait d'autres problèmes environnementaux, ouvre donc la voie à une controverse ${ }^{2}$ (Mounet, à paraître) où éthiques environnementales (Larrère, 1997), intérêts et valeurs (Weber, 1995) s'opposent. On peut cependant lister quatre grandes catégories d'impact concernant la plupart des sports de nature.

- Le piétinement est un impact commun à toutes ces activités, ne serait-ce que durant l'accès aux sites; il a donné lieu à de nombreuses publications dont certaines sont le fruit de protocoles rigoureux car le piétinement peut être normalisé grâce à des machines à piétiner dont les paramètres peuvent être finement ajustés.

- Le dérangement de la faune est également un thème assez général et porte sur des mammifères, des oiseaux et des poissons.

- Des modifications du recouvrement de la végétation et de la composition floristique ont pu être mises en évidence.

- A ces trois types d'impacts directs s'ajoute également toute une série d'impacts indirects liés aux accès motorisés (parkings, circulation automobile, etc.), relevant d'un aspect sanitaire (ordures, déchets organiques, atteinte à la qualité de l'eau) ou représentant même des nuisances purement anthropocentrées (paysage, bruit, etc.).

La gestion environnementale des sports de nature 1/ Des activités rejetées en espace protégé

Il faut noter en remarque liminaire, l'éviction qui a été à peu près systématique de certaines activités de ce type d'espaces, situation qui n'évolue que très peu. C'est le cas du VTT, simplement du fait qu'il est considéré comme un véhicule. Mais la pratique de l'équitation y a toujours été autorisée alors que la bibliographie montre qu'elle peut provoquer des dégâts sur le sol, surtout en zone humide, et sur la végétation (Edington \& Edington, 1986) qui sont plus importants que ceux dus au VTT (Price, 1985). La décision relève ici d'une simple application autoritaire des textes légaux (et probablement d'une certaine idéologie liée à la comparaison animal / machine...), sans tenir compte de la réalité de l'impact environnemental.

2/ Une gestion environnementale autoritaire ou concertée dans les espaces protégés à prérogatives réglementaires fortes

Deux Réserves naturelles confrontées à la gestion du Petit et du Grand Tétras sont une première illustration intéressante des types de gestion mises en œuvre. Ces deux galliformes, qui présentent une sensibilité particulière au dérangement hivernal, sont des espèces dont les populations font l'objet d'un suivi en raison d'une évolution très préoccupante.

La Réserve naturelle de la Haute chaîne du Jura a mis en place un plan de circulation pour la raquette à neige pour protéger le Grand Tétras après en avoir informé l'ensemble des acteurs concernés (Paget, 2003). Outre quelques erreurs dans la formulation, le plan a été 
perçu comme une prise de pouvoir du gestionnaire car les acteurs les plus concernés par l'activité (accompagnateurs, loueurs, divers prestataires) ainsi que certains acteurs locaux se sont trouvés écartés du processus décisionnel dont ils ont finalement subi la formalisation comme une gestion purement réglementaire. Aussi, cet état de fait s'est-il accompagné d'une mise en cause de la gestion et du gestionnaire dans une ambiance conflictuelle s'appuyant sur des interprétations différentes des comportements des oiseaux. Or, la volonté du gestionnaire était pourtant de bâtir un cadre de concertation... mais il s'est contenté de rester dans ce que Beuret considère comme la sphère des relations verticales (communication, information, consultation) sans entrer dans une concertation.

21 A l'opposé, la Réserve naturelle des Hauts plateaux du Vercors a formalisé et diffusé sa politique de fréquentation de loisir qui laisse une certaine liberté d'aller et venir aux randonneurs sans faciliter l'accès à l'espace par des balisages trop nombreux. Loin d'imposer aux prestataires de la raquette à neige un plan de circulation, le gestionnaire (le Parc naturel régional du Vercors) leur a proposé un «marquage » : la marque Parc de la Fédération des PNR leur est attribuée, ce qui contribue à leur promotion via les documents émis par le gestionnaire qui, de plus, associe un label de qualité à leur image. En compensation, les professionnels s'engagent notamment à éviter certaines zones d'hivernage du Tétras-lyre. Ce dernier aspect a donné lieu à un dialogue portant notamment sur la légitimité des demandes exprimées avant d'être accepté et pris en charge par les professionnels.

Ce type d'opération existe dans d'autres espaces protégés. L'espace fait, au minimum, la promotion des professionnels; il arrive même que des actions de formation et même d'aide à l'équipement (comme pour du matériel optique) soient engagées. En contrepartie, ces derniers s'engagent à respecter des prescriptions environnementales, à les expliquer à leurs clients, voire même à aider le gestionnaire pour le suivi de certaines espèces.

Une telle logique a été développée par le Parc de Port-Cros qui a formalisé une charte de la plongée et proposé un partenariat aux clubs et prestataires. Ces derniers s'engagent à limiter certaines pratiques pouvant mettre en péril l'environnement et acceptent d'augmenter les temps de rotation entre deux palanquées (ce qui, en évitant trop de turbidité, permet aussi une meilleure qualité de prestation). Les prestataires signataires de la charte battent pavillon de partenaire du Parc.

Une autre illustration concerne les Parcs nationaux des Ecrins et de la Vanoise qui ont signé des conventions pour diverses activités (escalade, vol libre, etc.) avec de nombreux partenaires (fédérations et prestataires sportifs, associations de protection de la nature, etc.) : l'objectif est, notamment, d'encadrer des activités qui peuvent avoir un impact environnemental et certaines négociations n'excluent pas de procéder à quelques échanges ponctuels entre sites des zones centrale et périphérique ${ }^{3}$, dans la mesure où ces derniers présentent un intérêt biologique particulier. Il en est de même pour la Réserve des Gorges de l'Ardèche qui a passé une convention avec les organes départementaux de la Fédération française de la montagne et de l'escalade pour gérer les sites de reproduction de rapaces: dans certains cas, l'accès à une falaise peut être donné au moment de la saison où il est avéré que la nidification n'a pas eu lieu. Mais cela suppose une concertation et un suivi permanents.

Ces derniers cas illustrent clairement la tendance actuelle qui consiste à mettre de côté, au moins pour un temps de négociation, les prérogatives réglementaires pour établir des 
partenariats sur la base minimale d'un dialogue voire même d'une gestion concertée qui a toujours pour logique des jeux à somme positive. Mais il est certain que ces négociations s'appuient sur l'autorité légale donnée par le cadre réglementaire de ces espaces qui laisse la possibilité à tout moment au gestionnaire de rompre la négociation et de revenir à une attitude autoritaire en cas de besoin. Cependant, si ces concertations sont engagées, c'est que le gestionnaire pense que les modalités adoptées permettent, en définitive, de rendre plus efficace la gestion environnementale.

3/ Parcs naturels régionaux et nature ordinaire : des médiateurs « faibles »

Mais d'autres espaces, dépourvus de prérogatives réglementaires fortes, expérimentent aussi des gestions concertées. Les Parcs Naturels Régionaux (PNR) sont de ceux-là. Les PNR du Vercors et du Massif des Bauges ont débuté depuis plusieurs années la rédaction de schémas des sports de nature, d'organisation pour le Vercors et de cohérence pour les Bauges. La gestion concertée touche tous les aspects de ces activités (accès et utilisation des sites, sécurité, équipement, etc.). Actuellement une action sur l'escalade est en cours dans les Bauges et met en interaction grimpeurs de tous statuts (guides, moniteurs d'escalade, Fédération de la Montagne et de l'escalade, Club alpin français), naturalistes, professionnels du tourisme et élus au sein du comité de pilotage autour du problème difficile des zones de reproduction des rapaces protégés. Aucune opposition ne s'est actuellement fait jour. Cependant, si la démarche adoptée a été très largement couronnée de succès pour les autres aspects du développement des sports de nature, il est difficile de prédire son efficacité dans le cadre d'une gestion environnementale.

Le département de l'Ardèche a également fait un travail considérable sur le Plan départemental des espaces, sites et itinéraires relatifs aux sports de nature. Devant les incertitudes liées aux impacts environnementaux des sports de nature, des enjeux environnementaux ont été décrits au niveau départemental et servent de base à l'évaluation environnementale des projets. Là encore, sportifs et protecteurs de la nature ont été en concertation pour élaborer et valider le référentiel, mais il est encore trop tôt pour porter un jugement définitif sur la situation.

Ces derniers cas, hors espace strictement protégé, ont en commun l'animation par des médiateurs qui ne possèdent aucun moyen réglementaire de pression. Ces médiateurs s'appuient plutôt sur leur " pertinence " (Friedberg, 1993) qui consiste à résoudre les problèmes des acteurs et, notamment, à les aider à collaborer et à trouver les subventions nécessaires à leurs projets. Cela suppose la construction d'un collectif associant diverses identités dont celles qui sont émergentes (Callon et al., 2003 ; Latour, 2006). Parmi elles, des actants non humains dont notamment les espaces, ont une importance centrale dans la négociation. Là, se joue le jeu de la légitimité de chacun pour faire partie de la concertation, selon les processus décrits par Beuret (2006). Le moteur est donc tout autre que dans le cas des espaces protégés à prérogatives réglementaires fortes, la " faiblesse " relative du médiateur faisant, en quelque sorte, sa force, à la condition qu'il soit suffisamment légitime pour les diverses parties en présence. Ces réflexions rejoignent en grande partie celles que Beuret et Trehet (2001) ont menées sur la concertation en espace rural.

Démocratie participative, médiateurs forts et faibles

Ces quelques exemples posent finalement au moins deux questions sur la gestion environnementale concertée des sports de nature, outre celle de la confrontation entre autorité et pouvoir: Comment fonctionne cette gestion? et est-elle une forme de 
participation et à quel niveau ? Bien que les situations présentées n'aient pas l'ambition de l'exhaustivité, elles permettent d'ébaucher quelques pistes de réflexion.

D'une part, elles présentent le paradoxe apparent de médiateurs usant de leur autorité légale pour amener les acteurs à une concertation. Mais il s'agit finalement plus d'un recours au "pouvoir » au sens organisationnel des analyses de Crozier et de Friedberg, c'est-à-dire la capacité à modifier le comportement de son protagoniste, car il est évident que le seul exemple de régulation uniquement réglementaire induit une situation conflictuelle. L'autorité est donc plutôt utilisée d'un point de vue stratégique en essayant de ne pas avoir à la mettre en œuvre. En revanche, les médiateurs « faibles » sont bien des traducteurs au sens donné dans les travaux de Callon et Latour dans la mesure où leur " traduction " permet aux divers acteurs concernés de créer un "réseau » (Callon, 1986; Callon et Law, 1989 ; Latour, 1984 et 2006). Et les exemples pratiques, conformément à la théorie de l'acteur-réseau, montrent bien que, pour que les jeux engagés soient à somme positive, il est indispensable que soit établies une confiance et une transparence ${ }^{4}$.

Mais si cette seconde alternative semble séduisante, elle pose au moins trois problèmes. Les deux premiers, relatifs à la gouvernementalité et à la gouvernabilité, sont posés par Theys (2003) dans sa critique de la gouvernance environnementale.

- Comment et sur quelle base sont élaborés les objectifs de ces médiateurs ? Existe-t-il une gouvernementalité de ces contextes d'action au sens de l'existence d'une visée politique autre que la justice par « avantage mutuel $»^{5}$ ? N'y a-t-il pas là également un risque de dérive technocratique dans la mesure où le technicien, bien que mandaté par des élus, a une marge de manœuvre assez considérable?

- Eu égard à la contingence forte de ces contextes d'action, la concertation engagée ne dépend-elle pas fondamentalement de la gouvernabilité de ceux-ci, c'est-à-dire de la capacité de leurs acteurs à résoudre les problèmes et donc de la complexité du système et des enjeux? Si cette critique s'avère fondée, il est à craindre que les situations les plus complexes ne puissent être traitées autrement que par le recours à l'autorité.

- Se pose également le problème de l'efficacité et de la pérennité de ce type d'action : que deviendrait le réseau sans le médiateur ? Il est douteux que les acteurs puissent accéder à une véritable autonomie si celui-ci se retire. Et on peut s'interroger sur la pérennité des dispositifs engagés dans la mesure où ils reposent sur des financements locaux. De plus, passée la première effervescence de la nouveauté de la concertation, les règles du jeu peuvent être naturalisées par les acteurs qui vont alors commencer à jouer sur celles-ci pour obtenir du pouvoir (Friedberg, 1993)6.

Enfin, Mermet (2001) distingue trois types de démocratie : élective, participative et celle des porte-parole. Callon et al (2001) considèrent que la démocratie élective, « délégative ", a pour conséquence de priver les citoyens de droit à la parole durant la durée du mandat de l'élu car, pour eux, les modalités de la participation sont à rechercher dans le double approfondissement du problème à traiter et du collectif formé d'identités qui peuvent être émergentes ${ }^{7}$.

Les divers exemples de gestion concertée qui ont été décrits ci-dessus renvoient à une démocratie des porte-parole. Le grand public ne participe pas à ces scènes locales de négociation, ce qui exclut une véritable gestion participative. Or, il existe un problème spécifique aux sports de nature : une part des publics concernés, variable selon les activités mais généralement très importante, est constituée de pratiquants indépendants ne relevant d'aucune organisation identifiable. Ces « indépendants » ou " inorganisés » (au sens purement organisationnel du terme) n'ont, par nature, aucun porte-parole. Il existe, 
certes, de multiples dispositifs pour " gérer » le public : éloignement des parkings, choix du mode de balisage, concentration ou diffusion des flux, etc. Ainsi en est-il sur les Hauts plateaux du Vercors où le balisage sert d'élément de régulation. A Port-Cros, un sentier sous-marin permet de concentrer le public dans une zone d'interprétation où le nourrissage du poisson le rend proche du nageur sous-marin. Flux de public, donc : le public sans porte-parole se réduirait-il à des flux ? Il n'existe, actuellement, aucune possibilité réelle d'engager une concertation durable avec ces « inorganisés » sur la gestion des espaces utilisés par les sports de nature : si, dans la station de ski, le public a la possibilité de s'exprimer a minima par son comportement de consommation, il n'en est rien dans des espaces libres d'accès et non payants. On retrouve ici la réflexion de Jean Viard $(2000,149)$ sur la mise en mobilité de notre société et ses conséquences sur la prise en compte des «nomades » contemporains dont font partie touristes et " post-touristes » : une " démocratie résidentielle » peut-elle assurer la représentation de ceux-là, du fait que " on vote plus souvent là où l'on dort que là où l'on agit "?

Les modalités d'une démocratie participative restent donc encore à inventer pour ce public. Rares sont les pistes intéressantes car il n'est pas forcément fidèle aux mêmes lieux : comment, dans ces conditions lui expliquer les enjeux environnementaux et comprendre les besoins de ces acteurs qui se révèlent en définitive " non pertinents " (Friedberg, 1993) puisqu'il est impossible de nouer avec eux une relation stratégique suivie?

Sports de nature et environnement : quel devenir?

Quelles sont les leçons à tirer des situations expérimentales étudiées ? Il est encore bien difficile de trancher définitivement en terme d'efficacité entre autorité et concertation. Mais une chose est sûre, le seul exemple de recours à l'autorité dans un espace protégé à prérogatives réglementaires s'avère un échec. Cela va de pair avec l'évolution actuelle de la protection de la nature qui admet maintenant le fait que l'Homme soit non seulement présent dans la nature, mais en plus qu'il ait la charge de la gérer pour maintenir la biodiversité des espaces protégés (Mougenot, 2002).

Ce qui caractérise toutes les autres situations est la présence d'un médiateur, qu'il soit faible ou fort. Mais si la présence d'un médiateur est possible dans des espaces gérés, elle ne va pas de soi dans la nature ordinaire. Dans ce cas, se pose la question du financement $\mathrm{du}$ processus participatif. C'est donc l'existence de politiques environnementales locales de la nature qui est à interroger en ce qui concerne l'avenir car la prise en compte de l'environnement dans la gestion des sports de nature est encore en construction: elle peut bénéficier de la reconnaissance légale de niveau départemental, des espaces, sites et itinéraires, mais celle-ci ne précise pas comment l'environnement doit être pris en compte dans le développement de ces activités. Or, un vaste mouvement d'équipement et de sécurisation des espaces sportifs est en cours du fait de la massification de ces activités. Une analogie permet d'éclairer les enjeux. Après la première guerre mondiale, Michelin fournissait aux automobilistes de véritables road books, très proches de ceux utilisés actuellement en VTT, qui les avertissaient des dangers comme un virage particulier ou une forte descente. La firme a également été à l'origine des panneaux routiers portant des informations directionnelles bien proches de celles de l'actuelle signalétique directionnelle en espace naturel. En se référant, à ce que sont devenues les routes françaises en quelques décennies et à l'évolution actuelle des sports de nature, il est à craindre, si cette évolution n'est pas régulée, que l'ensemble de la nature ordinaire 
ne se transforme, à terme, en un terrain de jeu entièrement banalisé, équipé, signalisé, où flore et faune n'auront plus aucune zone de refuge.

\section{BIBLIOGRAPHIE}

Beuret J.-E. et C. Trehet (2001) Pour la gestion concertée de l'espace rural : appuyer des médiations territoriales, Courrier de l'environnement, 43.

Beuret J.-E. (2006) La conduite de la concertation - pour la gestion de l'environnement et le partage des ressources, Paris, L'Harmattan.

Callon M., Lascoumes P ; Barthes Y. (2001). Agir dans un monde incertain : essai sur la démocratie technique, Paris, Seuil.

Callon M. (1986) Eléments pour une sociologie de la traduction, Année sociologique, XXXVI.

Callon M. et J. Law (1989) La protohistoire d'un laboratoire, In Callon M. (ed.) La science et ses réseaux, Paris, La Découverte.

Dahl R. A. (1957) The Concept of Power, Behavioral Sciences, 2.

Edington J.M. \& Edington M.A. (1986) Ecology, recreation, tourism, Cambridge University Press, Cambridge.

Friedberg E. (1993) Le pouvoir et la règle, Dynamiques de l'action organisée, Paris, Seuil,

Jacob G.R. et Schreyer R. (1980) Conflict in outdoor recreation : a theorical perpective, Journal of leisure research, 12(4), 368-380.

Kalaora B. (1993) Le Musée vert, Paris, L’Harmattan.

Kalaora B. (1998) Au-delà de la nature, l'environnement, L'observation sociale de l'environnement, Paris, L'Harmattan.

Larrère C. (1997) Les philosophies de l'environnement, Paris, P.U.F.

Latour B. (1984) Les microbes : guerres et paix, Paris, La Découverte.

Latour. B. (1991) Nous n'avons jamais été modernes. Paris. La Découverte.

Latour B. (2006) Changer de société - refaire de la sociologie, La Découverte, Paris.

Mermet L. (2001) L'institution patrimoniale du Haut Béarn : gestion intégrée de l'environnement ou réaction anti-environnementale? Annales des Mines, Janvier 2001.

Mermet L. (1992) Stratégies pour la gestion de l'environnement, La nature comme jeu de société ? Paris, L'Harmattan.

Mougenot, C. (2002) Prendre soin de la nature ordinaire, MSH, INRA.

Mounet J.-P. (1997) Les activités physiques et sportives dans l'offre touristique, Cahiers Espaces, 52, pp 102-110.

Mounet J.-P., Nicollet J.-P., Rocheblave M. (2000) L'impact des activités sportives de nature sur l'environnement naturel. Montagnes Méditerranéennes, 11 Tourisme sportif et territoires. 
Mounet J.-P., Mounet -Saulenc H., Paget E. (2004) Sports de nature et environnement. Elaboration d'un instrument d'évaluation environnementale pour le PDESI de l'Ardèche, Laboratoire SENS, Association Cohérence, Conseil général de l'Ardèche.

Mounet J.-P. (à paraître) Sports de nature, développement durable et controverse environnementale. Natures Sciences Sociétés.

Paget E. (2003) La raquette à neige dans la Haute-chaîne du Jura: un révélateur de la gestion problématique de la Réserve naturelle, DEA-STAPS Sport, Performance et Environnement Social.

Pociello C. (1981) La force, la grâce et les réflexes, In Pociello C. (ed.) Sports et société. Approche socio-culturelle des pratiques, Vigot, Paris.

Pociello C. (1995) Les cultures sportives, Presses Universitaires de France, Paris.

Price M. (1985) A review of research into the impacts of recreation on alpine vegetation in the Cairngorm mountains, Scotland, In Bayfield N.G. \& G.C. Barrow (ed.) The ecological impact of outdoor recreation on mountains areas in Europe and North America, R.E.R.G. Report $\mathrm{N}^{\circ} 9$.

Theys J. (2003) La Gouvernance, entre innovation et impuissance, Revue Développement Durable et Territoires, Dossier 2 : Gouvernance locale et Développement Durable

Viard J. (2000) Court traité sur les vacances, les voyages et l'hospitalité des lieux, La Tour d'Aigues, Editions de l'aube.

Vigarello G. (1981) D'une nature, l'autre : les paradoxes du nouveau retour in Pociello C. (ed.) Sports et société, Approche socio-culturelle des pratiques, Vigot, Paris.

Weber, M (1995). Economie et société, Pocket, Paris.

\section{NOTES}

1. Le ski bénéficie des dispositions de la Loi Montagne, tandis que les sentiers de randonnée peuvent donner lieu à l'établissement d'un Plan départemental des itinéraires de promenade et de randonnée.

2. Les controverses environnementales questionnent la prééminence des connaissances scientifiques dans les prises de décision et, de ce fait, mettent les acteurs dans l'obligation de gérer une incertitude scientifique sans recours possible à des conclusions irréfutables.

3. Devenues zones « cœur » et « d'adhésion ».

4. La traduction consiste, pour un acteur, à formuler le même problème de différentes façons de telle sorte que chacun des acteurs concernés trouve un intérêt à sa résolution. Les acteurs sont alors mis en coopération par le traducteur sous la forme d'un réseau. Callon et Law (1989), évoquant un cas clinique devenu exemplaire, ont montré que ce réseau ne peut exister qu'à la double condition que la confiance existe entre ses membres et que le traducteur fasse preuve de transparence dans ses actions.

5. Theys (2003) pointe le risque que peut faire courir une gouvernance environnementale sans visée politique spécifique : elle ouvrirait la voie à des arrangements avantageux entre les acteurs les mieux placés qui seraient susceptibles de n'équilibrer stratégiquement que leurs propres intérêts réciproques.

6. La sociologie organisationnelle considère que les règles formelles sont à la fois le fruit et un mode de structuration de l'action organisée. Quand des règles apparaissent, elles peuvent devenir l'objet d'un nouveau jeu entre les acteurs, car elles sont alors porteuses de nouvelles sources potentielles de pouvoir. 
7. Callon et al. (2003), reprenant la critique de la modernité que fait Latour (1991), considèrent que la démocratie élective présente un double inconvénient. Elle ne laisse pas la possibilité aux électeurs de formuler eux-mêmes les problèmes qu'ils voudraient voir résolus. Elle empêche leur expression entre deux élections, l'élu étant alors libre d'agir à sa guise. La gestion participative ne peut donc exister pour ces auteurs que si elle permet de donner la possibilité aux acteurs de redéfinir par eux-mêmes les problèmes et qu'elle autorise tous les acteurs concernés, même s'ils n'ont pas de porte-parole, à avoir accès au débat.

\section{RÉSUMÉS}

Cet article interroge les conséquences de la croissance des sports de nature dans leur rapport à l'environnement. Leur évolution récente pose le problème de leur durabilité vue sous l'angle de la préservation environnementale au sein d'espaces protégés comme de la nature ordinaire. La controverse portant sur leur impact environnemental semble exclure les solutions autoritaires. La mise en œuvre d'une démocratie participative est différente selon qu'il s'agit d'espaces protégés à prérogatives réglementaires fortes ou non ou de nature ordinaire, définissant des médiateurs forts ou faibles. Quoi qu'il en soit la participation reste limitée aux porte-parole en laissant de côté les très nombreux pratiquants hors de toute organisation.

This article interrogates the consequences of the growth of natural sports in their relationship to the environment. Their recent evolution raises the problem of their durability seen under the angle of the environmental conservation within spaces protected as within the common nature. The controversy concerning their environmental impact seems to exclude the authoritarian solutions. The implemented of a participative democracy is different as it is about spaces protected in strong statutory privileges or not, or about common nature, defining strong or weak mediators. However, the participation remains limited to the spokesmen by leaving aside the very numerous sport followers outside any organization.

\section{INDEX}

Mots-clés : démocratie participative, gestion environnementale, sports de nature, médiation Keywords : environmental management, Outdoor sports, participative democracy

\section{AUTEUR}

\section{JEAN-PIERRE MOUNET}

Jean-Pierre Mounet est Maître de conférences HDR en STAPS (sociologie du sport, du tourisme et des loisirs), Docteur en écologie, Directeur de l'IUP Loisir, Environnement, Sport, Tourisme (IUP LEST). Ses Thèmes de recherche portent sur sports de nature et tourisme durable, gestion environnementale, concertation, conflits d'usage. Laboratoire SENS (Sport et environnement social), Université Joseph Fourier - UFR APS, BP 53 - 38041 Grenoble Cedex 9 Laboratoire : 33 (4) 
76635089 (actuel), 33 (4) 76635044 (à partir de mi-septembre) jean-pierre.mounet@ujfgrenoble.fr 